\title{
Impacto de la rehabilitación vestibular en el riesgo de caída y la confianza del paciente
}

\author{
Impact of vestibular rehabilitation on the risk of falling and patient confidence
}

Ignacio Novoa $\mathbf{C}^{1}$, Tamara Aranda $\mathrm{R}^{2}$, Yarella Molina $\mathrm{B}^{2}$, Víctor Mercado $\mathrm{M}^{3}$.

\section{RESUMEN}

Introducción: Las alteraciones del sistema vestibular como la hipofunción vestibular unilateral, predisponen al usuario a sufrir caídas alterando así su confianza.

objetivo: Determinar el impacto de la rehabilitación vestibular en el riesgo de caídas y el grado de confianza en mujeres con hipofunción vestibular unilateral.

Material y método: Se realiza estudio prospectivo en 20 pacientes de género femenino mayores de 60 años con diagnóstico de patología vestibular periférica. Se evalúa el riesgo de caídas con escala Tinetti y el grado de confianza al realizar actividades de la vida diaria por medio de la escala ABC (Activities-specific Balance Confidence), antes y después de realizar terapia de rehabilitación vestibular (RV).

Resultados: Las dos variables estudiadas Tinetti y escala ABC demostraron mejoras significativas en la mayoría de las pacientes, demostrando que a medida que aumenta el grado de confianza disminuye el riesgo de caídas.

Conclusión: Los resultados obtenidos de este estudio sugieren que la terapia de RV es eficiente en mejorar el riesgo de caídas y favorecer el aumento de confianza en las actividades de la vida diaria en el grupo de pacientes estudiadas.

Palabras clave: Hipofunción vestibular unilateral, rehabilitación vestibular, riesgo de caídas, confianza.

\begin{abstract}
Introduction: Changes in the vestibular system, such as unilateral vestibular hypofunction, predispose the user to suffer falls, thus altering his confidence.

Aim: To determine the impact of vestibular rehabilitation on the risk of falls and the degree of confidence in women with unilateral vestibular hypofunction.
\end{abstract}

\footnotetext{
1 Departamento de Ciencias de la Rehabilitación, Escuela de Kinesiología, Universidad Santo Tomas, Viña del Mar, Chile.

2 Departamento de Ciencias de la Rehabilitación, Escuela de Kinesiología, Universidad Santo Tomás, Viña del Mar, Chile.

3 Instituto Neurobalance, Viña del Mar, Chile.
}

Los autores declaran no tener conflictos de interés.

Recibido el 22 de abril, 2019. Aceptado el 11 de mayo, 2019. 
Material and method: A prospective study was conducted in 20 female patients over 60 years of age with a diagnosis of peripheral vestibular pathology. The risk of falls with a Tinetti scale and the degree of confidence in carrying out activities of daily living are evaluated through the ABC scale (Activities-specific Balance Confidence), before and after performing vestibular rehabilitation therapy (VRT).

Results: The two variables studied, Tinetti and ABC scale showed significant improvements in most of the patients, demonstrating that as the degree of confidence increases the risk of falls decreases.

Conclusion: The results obtained from this study suggest that VRT is efficient in improving the risk of falls and favoring an increase in confidence in the activities of daily life in the group of patients studied.

Key words: Unilateral vestibular hypofunction, vestibular rehabilitation, risk of falls, confidence.

\section{INTRODUCCIÓN}

Los movimientos de la cabeza en diferentes direcciones son detectados y codificados por el sistema vestibular, éste nos permite además una adecuada estabilización y orientación de la postura corporal, definiendo la estabilización de la mirada por medio del reflejo vestíbulo-ocular'1. Es por ello que la disfunción de los aparatos visual, vestibular y somatosensorial y su respectiva integración al sistema nervioso central, desencadenará vértigo y otros síntomas asociados ${ }^{2,3}$. Los trastornos del sistema vestibular según la sociedad internacional de neurootología de Bárány se caracterizan por vértigo, mareo, alteraciones visuovestibulares y alteraciones posturales ${ }^{4}$. Estas alteraciones presentes en los pacientes afecta su equilibrio y estabilidad, aumentando por consiguiente el riesgo de sufrir caídas, lo que provoca miedo a deambular de manera independiente, es decir, generan un síndrome poscaída ${ }^{5}$. La definición más conocida y utilizada del síndrome poscaída es la de Tinetti quien la define como la pérdida de confianza en sí mismo para evitar las caídas durante la realización de actividades esenciales y relativamente no peligrosas que llevan al individuo a evitar las tareas que él es capaz de realizar-8.

Cuando el sistema vestibular periférico se daña de forma unilateral, la actividad neuronal aferente de los núcleos vestibulares del lado lesionado se reduce en comparación con la información aferente que ingresa a los núcleos vestibulares contralaterales lo cual es conocido como hipofunción vestibular unilateral (HVU). En un estudio realizado en
2017, se investigó a adultos mayores que sufren de hipofunción vestibular, determinando que las tareas más frecuentes realizadas en el momento de una caída fueron caminar (5\%), subir o bajar escaleras $(11 \%)$, cambios de postura $(9 \%)$ y tomar un baño $(6 \%)^{9}$. El proceso de envejecimiento normal del ser humano genera modificaciones en el aparato locomotor y vestibular que predisponen a sufrir caídas ${ }^{10}$; el costo promedio en el sistema de salud por un episodio de lesión por caída para personas de 65 años o más en Finlandia y Australia fue de US\$ 3.611, lo que equivale a $\$ 2.463 .785$ pesos chilenos ${ }^{11}$. Por este motivo es importante considerar que el riesgo de padecer una caída en conjunto con la pérdida de confianza pueden ser indicadores clínicos cuantificables para la orientación del médico al momento de dar el alta en un paciente con HVU.

\section{OBJETIVO}

El objetivo de este estudio es determinar el impacto de la rehabilitación vestibular en el riesgo de caídas y en el grado de confianza en mujeres mayores de 60 años con diagnóstico de hipofunción vestibular unilateral.

\section{MATERIAL Y MÉTODO}

El alcance de este estudio es de tipo exploratorio ya que se busca ilustrar por qué ocurre un fenómeno, en qué condiciones se manifiesta y si se correlacionan. 
Nuestro diseño es de tipo preexperimental ya que no existe manipulación de la variable independiente ni grupo de control, solo se tomarán datos iniciales y finales de una terapia de rehabilitación realizada por un profesional kinesiólogo en su práctica clínica. Esta investigación fue revisada y aprobada por el Comité de Ética regional centro-norte de la Universidad Santo Tomás.

Los criterios de inclusión fueron mujeres mayores de 60 años, presencia de HVU (de acuerdo al primer documento de consenso del Comité para la Clasificación de los Trastornos Vestibulares de la Sociedad de Bárány ${ }^{12}$ ) y un puntaje de escala de Tinetti menor a 24 puntos $^{6}$. Los sujetos de estudio fueron en número de 20 , atendidas en el Centro Marina Médica de Viña del Mar durante el año 2018. La muestra tuvo un promedio de edad de 72,4. Del total, 11 presentaron HVU de lado izquierdo, y $9 \mathrm{HVU}$ de lado derecho. Los criterios de exclusión fueron la movilidad reducida 0 ausente según escala ETADI Etapa 5 subetapa A -que se refiere a la alteración en la deambulación ya sea con 0 sin ayuda técnica 0 asistencia del paciente-, que el paciente no pueda comprender órdenes simples, la presencia de dolor incapacitante según escala verbal análoga, el uso de prótesis o antecedentes traumáticos de miembro inferior que pudieran alterar la marcha.

Para evaluar el riesgo de caídas se utilizó la escala de Tinetti $^{6}$ la cual evalúa el equilibrio estático-dinámico y la marcha. Esta escala mide equilibrio en primera instancia con ojos cerrados, sentado, en bipedestación, realizando un giro de $360^{\circ}$ y con estímulo desestabilizador clasificando el comportamiento del paciente en base a puntos. En segunda instancia mide marcha y cuánto se demora de un punto a otro antes establecidos, la simetría, longitud del paso, entre otros lo que nos arrojará como resultado en conjunto los siguientes parámetros:

- menor a 19 puntos indica riesgo alto de caídas

- entre 19-24 puntos indican riesgo de caídas

- mayor a 24 puntos sin riesgo de caídas

En la medición del grado de confianza se utilizó el instrumento ABC (Activities-specific Balance and Confidence Scale $)^{13}$, esta escala de confianza y equilibrio en las actividades específicas consta de un cuestionario subjetivo aplicado al paciente, que refleja la confianza que siente para ejecutar ciertas tareas donde se va asignando un porcentaje de 0 a 100, donde 0 es nada de confianza y 100 es el máximo grado de confianza, lo cual nos arroja los siguientes parámetros:

- Un porcentaje $>80 \%$ indica un alto nivel de funcionamiento.

- Un porcentaje entre 50\%-80\% indica nivel moderado de funcionamiento.

- Un porcentaje $<50 \%$ indica bajos niveles de funcionamiento.

- Un porcentaje $<67 \%$ sugiere un riesgo sustancial de caídas.

La RV se realizó de manera individual según el requerimiento del paciente y de las alteraciones generadas por la patología. En esta investigación la RV constó de 5 sesiones, de una hora de duración y en días consecutivos. Los datos obtenidos en las variables Tinetti y $A B C$ antes y después de una RV se analizaron estadísticamente para determinar si se lograba disminuir el riesgo de caídas y aumentar el grado de confianza en las pacientes, además de correlacionar estas variables para valorar aún más su significancia. El análisis estadístico se llevó a cabo con el programa Graphpad Prism 5, el cual también sirvió para evaluar la distribución normal de la muestra, aplicando Shapiro Wilk, mientras que para determinar la correlación entre nuestras variables se utilizó $R$ de Pearson. Para graficar el riesgo de caídas y el grado de confianza se elaboraron gráficos de columnas mientras que para las correlaciones se utilizó gráficos de dispersión.

\section{RESULTADOS}

Respecto a la medición del riesgo de caídas por medio de escala de Tinetti, se consideró el promedio del puntaje previo y posterior a la RV, que fue de 20,3 y 24,5 puntos respectivamente (Figura 1), se observa el aumento del puntaje en la escala, con una diferencia de 4,2 puntos. Según estos valores las pacientes pasaron de un riesgo de caídas a sin riesgo de caída.

Al desglosar esta escala en sus ítems de marcha (Figura 2) y equilibrio (Figura 3), se determinó que la tendencia es a la mejora del equilibrio, que aumenta de un promedio de 11,3 a 13,8 por sobre 


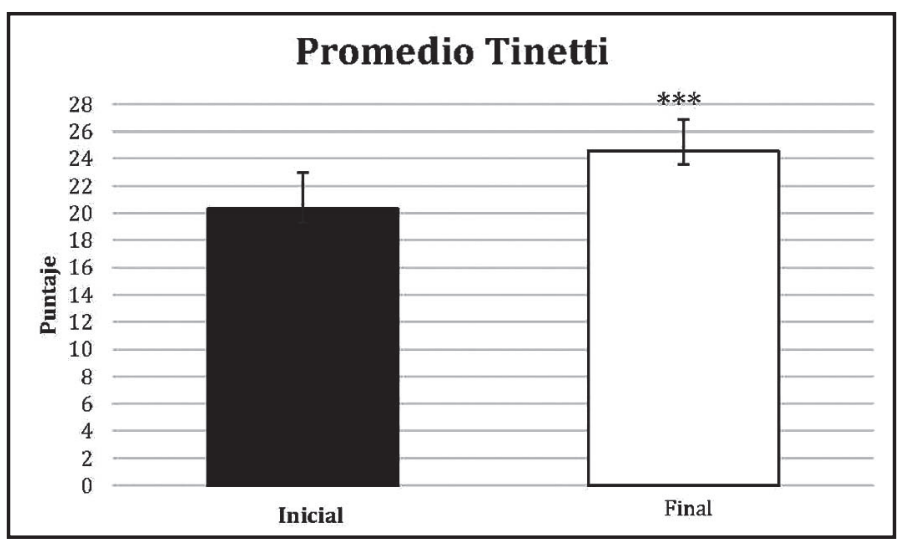

Figura 1. Promedio puntaje inicial y final a la rehabilitación. En barra negra se muestra la media del puntaje total de la escala Tinetti previo a la rehabilitación y en la barra blancas el promedio del puntaje total posterior a la rehabilitación. ***: Valor de $p=<0,0001$.

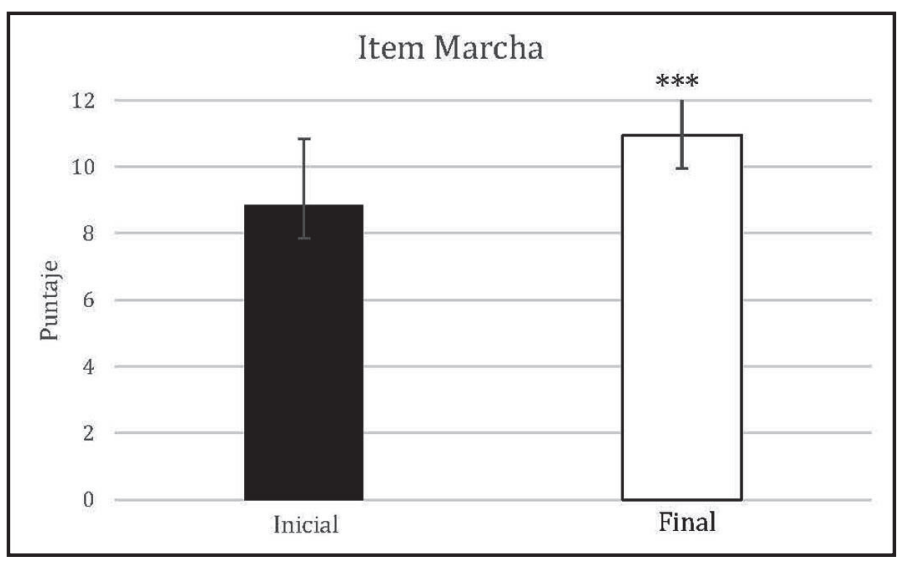

Figura 2. Promedio ítem marcha de escala Tinetti. Barra negra muestra el puntaje previo y barra blanca denota el puntaje promedio posterior a la rehabilitación vestibular. ${ }^{* * *}$ : Valor de $p=0,0001$.

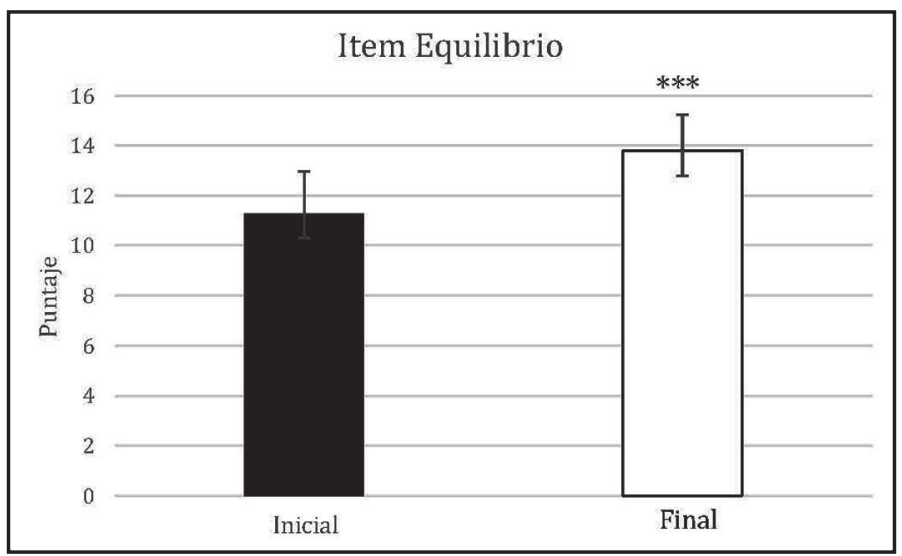

Figura 3. Promedio ítem equilibrio de escala Tinetti. Barra negra muestra el puntaje previo a la rehabilitación vestibular y la barra blanca denota el puntaje promedio posterior a la rehabilitación. ${ }^{* * *}$ : Valor de $p=<0,0001$. 
el promedio de marcha que fue desde 8,85 a 10,95 puntos.

En la medición de la segunda escala aplicada, $A B C$ Scale, que igualmente se realizó previo y posterior a la RV (Figura 4), el promedio inicial fue de $72 \%$ de confianza, lo que indica un nivel moderado de funcionalidad, donde 9 pacientes obtuvieron menos de $67 \%$ lo que indica un riesgo sustancial de caídas; en cuanto al promedio final, fue de $83 \%$ pasando de funcionalidad moderada, a una alta funcionalidad, aumentando en el promedio $12 \%$.

En cuanto a la correlación previa entre Tinetti y escala $A B C$ (Figura 5), se demostró que a medida que disminuye el riesgo de caídas, aumenta el grado de confianza, con un valor de $p<0,0001$, un valor de $R^{2}$ de 0,6256 . Donde los valores iniciaban

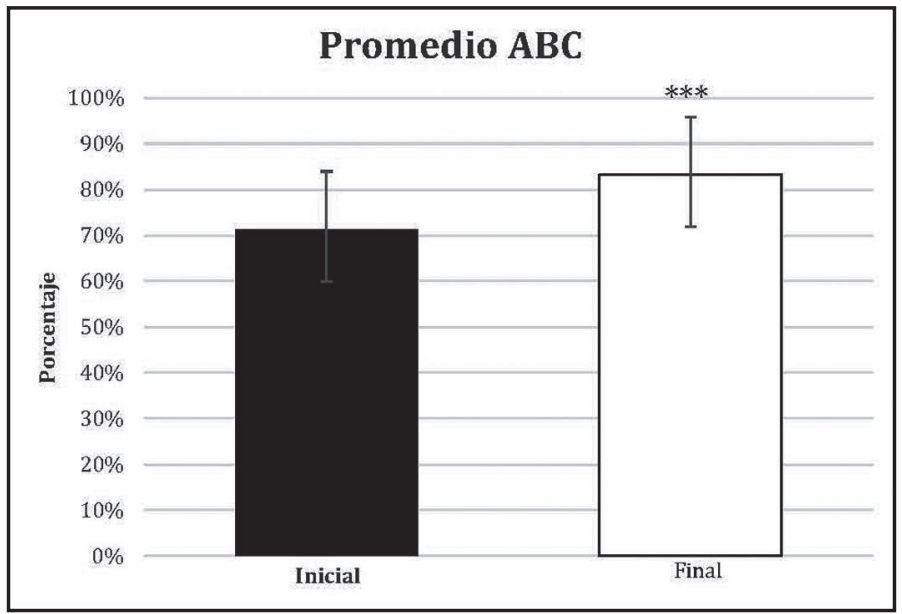

Figura 4. Promedio puntaje $A B C$ inicial y final a la rehabilitación. En barra negra se muestra la media del puntaje total de la escala $A B C$ previo a la rehabilitación y en la barra blanca el promedio del puntaje total posterior a la rehabilitación. ${ }^{* \star *}$ : Valor de $p=<0,0001$.

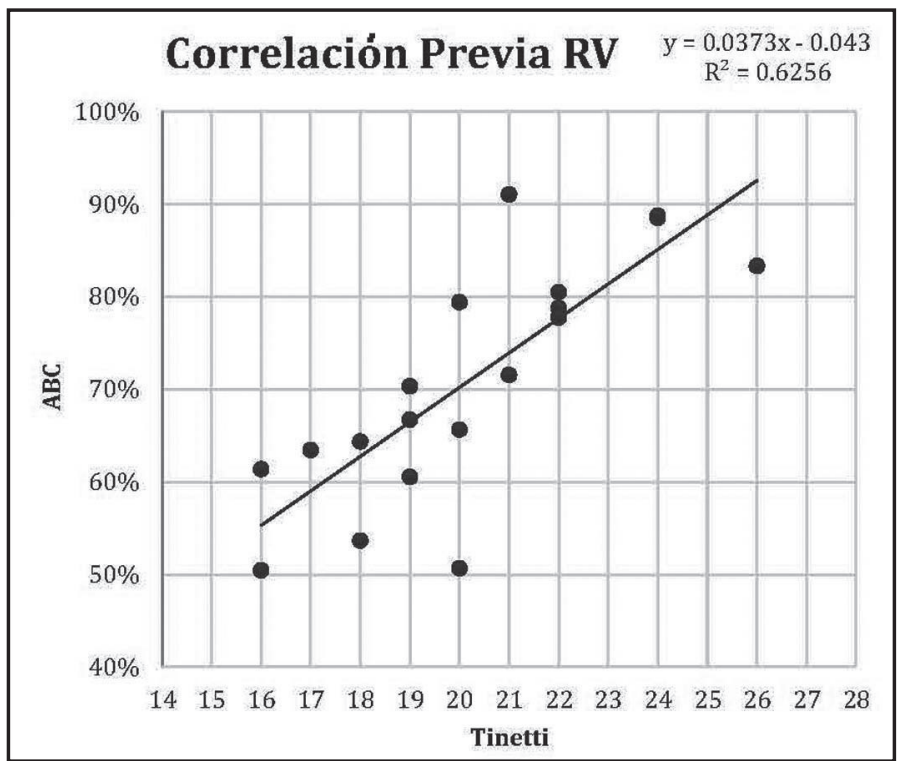

Figura 5. Gráfico de dispersión entre las escalas Tinetti y ABC previo a la rehabilitación vestibular. 


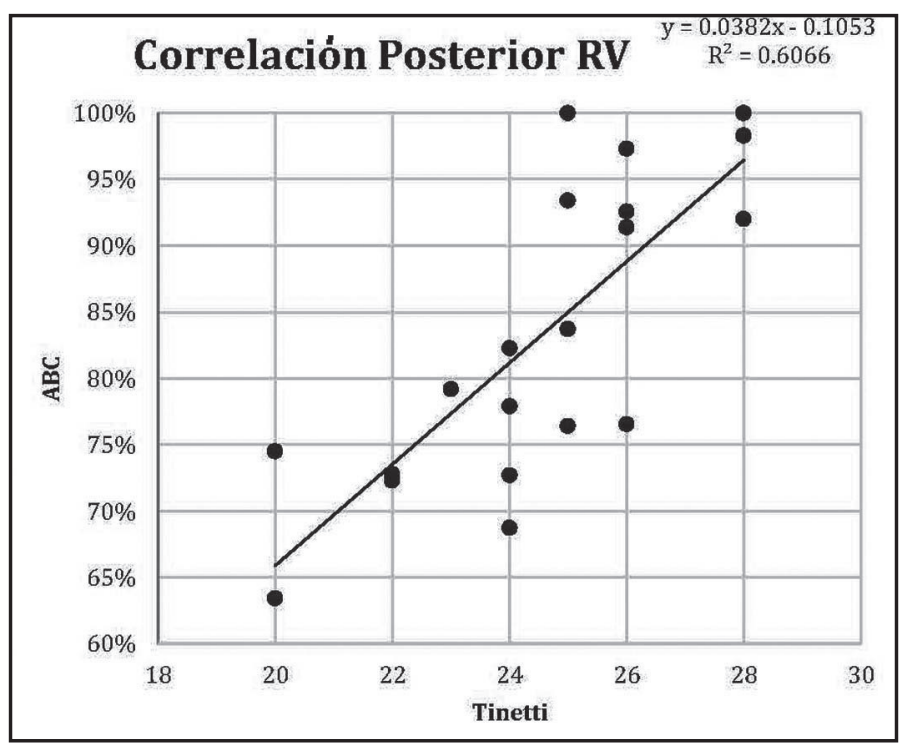

Figura 6. Gráfico de dispersión entre las escalas Tinetti y ABC posterior a la rehabilitación vestibular.

en $50 \%$ para escala $A B C$ y un Tinetti 16 puntos. Por otro lado, en la correlación posterior entre Tinetti y escala ABC (Figura 6), se demostró que a medida que disminuye el riesgo de caídas aumenta el grado de confianza, con un valor de $p<0,0001$ y un valor de $R^{2}$ de 0,6066 . En segunda instancia los valores inician sobre $65 \%$ en escala $A B C$ y un 20 de puntuación para Tinetti.

\section{DISCUSIÓN}

La RV demostró cambios significativos en el riesgo de caídas y el grado de confianza en el grupo de pacientes evaluadas a través de las pruebas Tinetti y escala ABC. Hasta el año 2007, se consideró que la RV generaba cambios en la condición vestibular del paciente en un periodo de 6 semanas con 1 02 sesiones de control semanales en promedio. Actualmente, este concepto ha cambiado ya que se ha comprobado que 5 sesiones la RV es efectiva en generar una disminución del riesgo de caídas y la autopercepción de discapacidad de pacientes con HVU ${ }^{14}$. Esta intervención mejora la estabilidad postural y la agudeza visual durante el movimiento de la cabeza reduciendo así el riesgo de caídas.
El enfoque de esta rehabilitación en este tipo de pacientes consiste en ejercicios motores óculovestibulares, los cuales fortalecen intensivamente los reflejos vestíbulo-oculomotores, vestíbulocerebelosos y vestíbulo-espinales, con lo cual se recupera gran parte de las actividades del diario vivir, lo que es muy valorado por el paciente. Según la guía clínica de rehabilitación vestibular establecida por el APTA en el año 2016, se evidencia que estos ejercicios deben ser acordes a los objetivos específicos establecidos al inicio de la evaluación, por lo que deben variar según el requerimiento de cada paciente ${ }^{15}$.

El año 1986 la doctora Mary Tinetti16, define la escala que lleva su nombre, la cual ofrece una ventaja sobre las otras evaluaciones musculares, ya que realiza una valoración tanto de la marcha como del equilibrio y estos dos aspectos brindan una información más completa para evaluar el riesgo de caída, determinando así si existen alteraciones en la marcha y en el equilibrio que requieren intervención, y valorar la presencia de posibles trastornos neurológicos o músculo esqueléticos. En estudios más recientes, se ha evaluado el riesgo de caídas con pruebas como el timed up and go $(\mathrm{TUG})^{16} \mathrm{y}$ el índice dinámico de marcha (DGI) ${ }^{17}$. Se ha mostrado 
eficacia de estas pruebas para evaluar el riesgo de caídas en pacientes con HVU, mas no existe gran evidencia de la utilización de la escala Tinetti para predecir caídas en pacientes con igual diagnóstico.

El aumento del riesgo de caídas genera una alteración en la confianza del paciente, diversos autores lo han establecido, Arfken y cols $^{18}$ la definen como un trauma psicológico que lleva a la persona a permanecer en su casa y a restringir sus actividades, lo cual la conduce a la declinación física. Para Howland y $\operatorname{cols}^{19}$ se trata de temor anormal caracterizado por una disminución de sus actividades. Existen numerosos instrumentos de medición para evaluar la confianza del paciente, en esta investigación se destaca el uso de la escala ABC, la que se ha utilizado desde 1993. Powell y Myers $^{20}$ correlacionaron la escala de eficacia de caídas (FES) con la escala ABC. Se determinó que eran consistentes y demostraron buena confiabilidad, convergencia y validez de criterio. En esta comparación se manifiesta que la escala FES se centra en la capacidad para evitar caídas evaluando actividades de la vida diaria poco arriesgadas, y su aplicación se ve dificultada en ancianos con bajo nivel educativo, no pudiendo aplicarse a todas las poblaciones; mientras que la escala $A B C$ evalúa la confianza en el equilibrio incluyendo tareas motrices más precisas ${ }^{21}$. Se ha evidenciado que la

\section{BIBLIOGRAFÍA}

1. Aedo C, Collao JP, Délano P. Anatomía, fisiología y rol de la corteza vestibular. Rev Otorrinolaringol Cir Cabeza Cuello 2016; 76: 337-46.

2. Velayos J, Diéguez G. Anatomía y fisiología del sistema nervioso central. CEU 2015; 5: 121-7.

3. Herdman S, Timothy C, Hain MD, Janet H. Vestibular Rehabilitation. CPR 2014; 1: 2-12.

4. Bisdorfia A, Breverni M, Thomas T. Classification of vestubular symptoms: Towards an international classification of vestibular desorders. J Vestib Res 2009; 19: 1-13.

5. Cruz E, Gonzales M, Lopez M, Godoy I, Perez M. Caídas: revisión de nuevos conceptos. HUPE 2014; 13: 86-95.

6. Tinetti ME, Richman D, Powell L. Falls efficacy as mayor capacidad de respuesta a la escala $A B C$ la hace más adecuada para detectar la pérdida de la confianza en el equilibrio en personas de la tercera edad con mayor rendimiento ${ }^{20}$. Anteriormente se han relacionado escalas que evalúan el riesgo de caídas con la escala $A B C$, no así, con la escala Tinetti como se ha elaborado en esta investigación, ya que se busca evidenciar la correlación existente entre estas dos escalas por lo ya mencionado a lo largo de este estudio. Finalmente, consideramos que la RV es una terapia efectiva en lograr los objetivos planteados, consideramos importante ser explícitos con los pacientes respecto a la forma de aplicar esta intervención y en las expectativas de los resultados.

\section{CONCLUSIÓN}

Esta investigación clínica demostró que posterior a realizar RV al grupo de pacientes con HVU, las puntuaciones obtenidas en las escalas de Tinetti y $A B C$ precisaron una mejora sostenida en sus ítems de marcha y equilibrio y un aumento en la funcionalidad respectivamente. Estos resultados concuerdan con la hipótesis inicial, que la RV disminuye el riesgo de caída y aumenta el índice de confianza. a measure of fear of falling. J Geronto/ 1990; 45: 239-43.

7. Tinett। ME, Baker D, King M. Effect of Dissemination of Evidence in Reducing Injuries from Falls. New Engl J Med 2008; 359: 252-61.

8. TinetTI ME, Kumar C. The patient who falls "It's always a trade-off". JAMA 2010; 303: 258-66.

9. Swanenburg J, Bäbler E, Adelsberger R, Straumann $D$, Bruin E. Patients with chronic peripheral vestibular hypofunction compared to healthy subject's exhibit differences in gaze and gait behavior when walking on stairs and ramps. PLoS One 2017, 12: 1-18.

10. Fernandez L, Breinbauer AH and Delano PH. Vertigo and Dizziness in the Elderly. Front Neurol 2015; 6: 144 . 
11. OMS. WHO Global Report on Falls Prevention in Older Age. Francia: WHO. Obtenido de Ageing and Life Course, Family and Community Health. 16 de Enero de 2018.

12. Bisdorff A, Von Brevern M, Lempert T, NewmanTOKER DE. Classification of vestibular symptoms: Towards an international classification of vestibular disorders. J Vestib Res 2009; 19: 1-13.

13. Cleary K, Skornyakov E. Predicting falls in community dwelling older adults using the Activities-specific Balance Confidence Scale. Arch Gerontol Geriatr 2017; 72: 142-5.

14. Novoa I, Donoso S, Martínez Y, Mercado A, Pino C, Mercado V. Efectividad de cinco sesiones de rehabilitación vestibular en mujeres mayores de 60 años con hipofunción vestibular. Rev Otorrinolaringol Cir Cabeza Cuello 2018; 78 : 259-66.

15. Riveros H, Correa C, Anabalón, Jl, Anaís JC. Efectividad de la rehabilitación vestibular en una serie clínica. Rev Otorrinolaringol Cir Cabeza Cuello 2007; 67: 229-36.

16. Rodríguez C, Lugo H. Validez y confiabilidad de la Escala de Tinetti para la población Colombiana. Revista de Asociación Colombiana de Reumatología 2012; 19: 218-33.

17. Verdecchia D, Mendoza M, Sanguineti F. Resultados tras la rehabilitación vestibular y terapia $\mathrm{Wii}^{\circledR}$ en pacientes con hipofunción vestibular unilateral crónica. Acta Otorrinolaringologica Española 2014; 65: 327-86.

18. Arfken CL, Lach HW, Birge SJ, Miller JP. The prevalence and correlates of fear of falling in elderly persons living in the community. $A m \mathrm{~J}$ Public Health 1994; 84: 565-70.

19. Howland J, Lachman ME, Peterson EW, Cote J, Kasten L, Jette A. Covariates of fear of falling and associated activity curtailment. Gerontologist 1998; 38: 549-55.

20. Powell LE, Myers AM. The Activities-specific Balance Confidence (ABC) Scale. Gerontol A Biol Sci Med Sci 1995; 50: 28-34.

21. Curcio C, Gómez Montes F. Temor a caer en ancianos: controversias en torno a un concepto y a su medición. Hacia la Promoción de la Salud 2012; 17: 186-204. 